\title{
Localised pulmonary arteritis in rheumatoid disease
}

\author{
JG ARMSTRONG, RH STEELE \\ From the Departments of Respiratory Medicine and Pathology, Princess Alexandra Hospital, \\ Brisbane, Australia
}

Patients with rheumatoid disease may develop thoracic complications, including pleural effusion, solitary or multiple necrobiotic nodules, pulmonary fibrosis, and Caplan's syndrome. ${ }^{1-3}$ Although vasculitis occurs in rheumatoid disease in a number of sites, pulmonary vasculitis has received little attention in the literature. We describe a case of acute, localised, necrotising pulmonary arteritis in a patient with rheumatoid disease.

\section{Case report}

A 58-year-old housewife was diagnosed as having rheumatoid arthritis in 1967 . She subsequently developed painful joint deformities and in June 1980 her left hip was replaced with a Müller prosthesis.

The patient was admitted to hospital on 30 September 1980 , complaining of right-sided pleuritic chest pain and mild dyspnoea for one week. Physical examination revealed that she was afebrile, blood pressure was $110 / 60$, and a systolic murmur was present at the cardiac base. A pleural friction rub was present over the right lower lobe and inspiratory crepitations were present over both lower lobes. Her hands showed the classical deformities of rheumatoid arthritis but no subcutaneous nodules or finger clubbing were present.

A chest radiograph (fig 1) showed a localised area of homogeneous consolidation involving the posterior segment of the right upper lobe. Reticular shadowing was present in the left lower lobe and had remained unchanged since 1977. An ECG showed T-wave inversion in leads V2 to V6. Other investigations were: haemoglobin $13 \cdot 1$ $\mathrm{g} / \mathrm{dl}$; white cell count $10.3 \times 10^{9} / 1$; ESR $95 \mathrm{~mm} / \mathrm{hr}$. Rheumatoid factor was present in a titre of $1 / 5120$. Antinuclear factor was not present.

A provisional diagnosis of pulmonary embolism with infarction was made and treatment with heparin was started. However, two weeks after the start of treatment the patient developed severe central chest pain, increasing dyspnoea and hypotension, and died.

\section{NECROPSY FINDINGS}

Significant disease was confined to the joints, heart, and lungs. Severe rheumatoid arthritis was confirmed. The heart weighed $300 \mathrm{~g}$ and neither ventricle was dilated or hypertrophied. There was a postero-lateral subendocardial

Address for reprint requests: Dr JG Armstrong, Department of Respiratory Medicine, Princess Alexandra Hospital, Woolloongabba, Qld 4102, Australia.

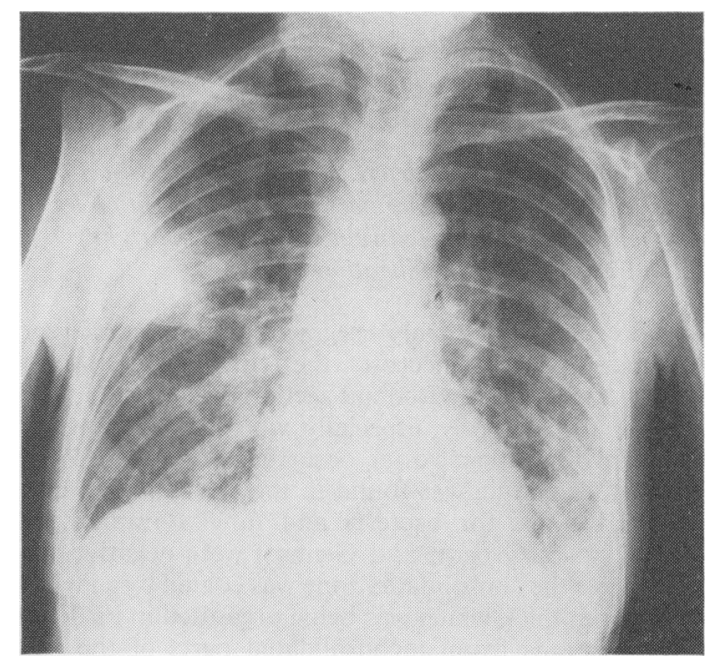

Fig 1 Chest radiograph showing consolidation in the posterior segment of the right upper lobe and reticular shadowing in the left lower lobe.

infarct aged about 10 days covered by fibrinous pericarditis. The coronary arteries were atherosclerotic and the aortic valve cusps were thickened.

The left lung was bound to the chest wall by dense fibrous adhesions. There was irregular interstitial fibrosis of the left lower lobe. No consolidation was present. Histologically the vessels were unremarkable and there was no evidence of pulmonary hypertension.

The right pleural space contained $300 \mathrm{ml}$ of turbid, blood-stained fluid and fibrinous adhesions were present posteriorly and in both fissures. The posterior part of the upper lobe of the right lung and the adjacent part of the apex of the lower lobe were consolidated, grey coloured and of dry texture. Histologically this area showed alveolar filling by macrophages and clumps of dense hyaline material undergoing early organisation. Polymorphonuclear leucocytes were not present except focally around damaged arteries. Many, but not all the pulmonary arteries in the consolidated area were affected by an acute necrotising arteritis. This did not affect vessels of external diameter less than $150 \mu \mathrm{m}$ or more than $400 \mu \mathrm{m}$. The veins and bronchial arteries were spared. The walls of the affected arteries showed fibrinoid necrosis, dissolution of the elastic lamina, and infiltration by polymorphs which extended a little way into the sur- 


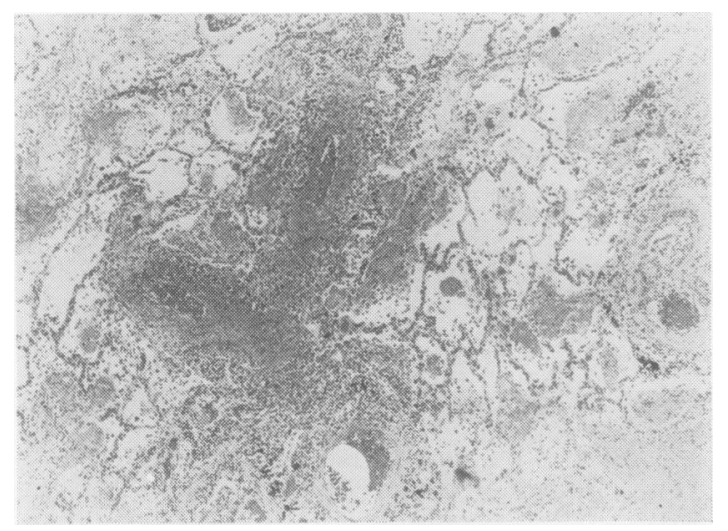

Fig 2 Two pulmonary arteries, each about $250 \mu \mathrm{m}$ in diameter, showing fibrinoid necrosis and infiltration by polymorphs. An unaffected vein is seen on the right. $(H$ and $E$, original magnification $\times 20)$.

rounding lung (fig 2). Only a segment of the circumference of some vessels was necrotic, the remainder of the wall being normal. In longitudinal sections necrosis could be seen to stop abruptly, especially where a junction with a larger or smaller vessel occurred. A solitary $5 \mathrm{~mm}$ rheumatoid nodule was found in the consolidated area.

Special stains for bacteria and fungi (Gram, ZiehlNielsen, PAS, Grocott and Giemsa) were negative. The pleura over the consolidated zone was coated by a layer of fibrin $2 \mathrm{~mm}$ thick which was being organised in its deeper levels. Sections from unconsolidated parts of the right lung did not show any arteritis and were otherwise unremarkable. There was no thrombus in any dissectable branches of the pulmonary artery.

No other organs showed any arteritis; by an oversight kidney sections were not taken but they were macroscopically normal.

\section{Discussion}

Acute fibrinoid arteritis localised to one area of the lung has not been described previously as a manifestation of

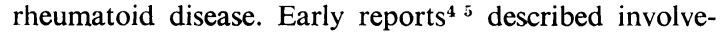
ment of both lungs with associated interstitial fibrosis. A later report ${ }^{6}$ described pulmonary infiltrates in patients with rheumatoid disease which cleared with corticosteroid treatment but lung biopsy proof of vasculitis was lacking. The reason for involvement of this area of lung is unknown but it may be the result of a local immunological phenomenon.

A feature of this case is the selective size of the vessels affected. Only arteries of external diameter between 150 and $400 \mu \mathrm{m}$ were involved. Fibrinoid arteritis involving vessels of a similar size (100 to $500 \mu \mathrm{m})$ was described previously in a patient with rheumatoid disease and pulmonary hypertension, " but the arteritis was considered to be secondary to the effects of pulmonary hypertension. There was no clinical or pathological evidence of pulmonary hypertension in the case that we have reported.

Acute pulmonary arteritis is uncommon in rheumatoid disease but should be considered in patients who develop localised pulmonary infiltrates. Lung biopsy should be considered to establish the diagnosis because the arteritis may respond to steroid or immunosuppressive therapy and therefore needs to be differentiated from pulmonary infection or infarction which occur more commonly in these patients.

\section{References}

${ }^{1}$ Macfarlane JD, Dieppe PA, Rigden BG, Clark TJH. Pulmonary and pleural lesions in rheumatoid disease. Br J Dis Chest 1978;72:288-300.

${ }^{2}$ Spencer-Jones J. An account of pleural effusions, pulmonary nodules and cavities attributable to rheumatoid disease. Br J Dis Chest 1978;72:39-56.

${ }^{3}$ Gardner DL. The pathology of rheumatoid arthritis. London: Edward Arnold, 1972:137-43.

'Ellman P, Ball RE. "Rheumatoid disease" with joint and pulmonary manifestations. $\mathrm{Br} \mathrm{Med} J$ 1948;2:816-20.

${ }^{5}$ Price TML, Skelton MO. Rheumatoid arthritis with lung lesions. Thorax 1956;11:234-40.

${ }^{6}$ Beck ER, Hoffbrand BI. Acute lung changes in rheumatoid arthritis. Ann Rheum Dis 1966;25:459-62.

' Kay JM, Banik S. Unexplained pulmonary hypertension with pulmonary arteritis in rheumatoid disease. $\mathrm{Br} \mathrm{J} \mathrm{Dis}$ Chest $1977 ; 71: 53-9$. 